\title{
Case Report of a Patient with a Rare and Life- Threatening Disorder: Thrombotic Thrombocytopenic Purpura
}

\author{
Svetlana Stankovikj
}

\begin{abstract}
Thrombotic thrombocytopenic purpura (TTP) is a syndrome that consists of the pentad of thrombocytopenia, microangiopathic hemolytic anemia, neurologic abnormalities, fever and renal disease. It was first described by Moschowitz in 1925 as a disease characterized by the pathological findings of hyaline thrombi in many organs. The blood protein von Willebrand factor ( $v W F$ ) has a central role in promoting platelet adhesion and aggregation. The enzyme ADAMTS13 (a metalloprotease) constitutively cleaves large VWF multimers that are secreted by the endothelium into shorter strands. In many cases, it has been identified as a genetic problem due to an irregularity in several suspect genes. In other cases, the disease appears out of nowhere and this is called an "idiopathic" cause. In acquired TTP, an autoantibody inactivates the ADAMTS13 protease, and thus the VWF multimers remain large and abundant. The multimers bind platelets and form aggregates in the microvasculature that induce thrombus formation. The micro thrombi cause tissue ischemia, platelet consumption, and microangiopathic hemolytic anemia. Brain involvement is common and leads to stroke, seizure, confusion, and headache. Renal injury occurs in a minority of patients, and it is usually modest. The syndrome rarely present with the pentad of symptoms. . Currently, unexplained thrombocytopenia and microangiopathic hemolytic anemia are the two criteria required to establish the diagnosis. Prompt initiation of the management is an important factor in improved patient survival.
\end{abstract}

Keywords: thrombotic thrombocytopenic purpura (TTP), hemolytic uremic syndrome (HUS), von Willebrand factor (vWF), ADAMTS13, plasma exchange

\section{Introduction}

Thrombotic microangiopathies are a group of hereditary and acquired syndromes with diverse mechanisms that lead to shared clinicopathological features: microangiopathic hemolytic anemia, thrombocytopenia, and organ injury (1). Thrombotic thrombocytopenic purpura (TTP) was first described by Moschowitz in 1925 as a disease characterized by the pathological findings of hyaline thrombi in many organs (2). In its classic form, it consists of the pentad of thrombocytopenia, microangiopathic hemolytic anemia, neurologic abnormalities, fever and renal disease. The blood protein von Willebrand factor ( $\mathrm{vWF}$ ) has a central role in promoting platelet adhesion and aggregation. The enzyme ADAMTS13 (a metalloprotease) constitutively cleaves large VWF multimers that are secreted by the endothelium into shorter strands (3). In many cases, it has been identified as a genetic problem due to an irregularity in several suspect genes (4).In these cases, there appears to be strong inherited tendencies. In other cases, the disease appears out of nowhere and this is called an "idiopathic" cause (5). In acquired TTP, an autoantibody inactivates the ADAMTS13 protease, and thus the VWF multimers remain large and abundant. The multimers bind platelets and form aggregates in the microvasculature that induce thrombus formation. The micro thrombi cause tissue ischemia, platelet consumption, and microangiopathic hemolytic anemia. Brain involvement is common and leads to stroke, seizure, confusion, and headache. Renal injury occurs in a minority of patients, and it is usually modest. Fever may develop but it is often due to precipitating infection (6).

Since, patients rarely present with the pentad of symptoms, prompt initiation of the management is an important factor in improved patient survival. Currently, unexplained thrombocytopenia and microangiopathic hemolytic anemia are the two criteria required to establish the diagnosis. A simple blood test will show shattered red blood cells and a low platelet counts, a definite sigh to initiate treatment (7).Plasma exchange is the standard of care for the initial management of acquired TTP (8). Plasma exchange is continued daily until resolution of organ dysfunction and stable normalization of the platelet count is obtained. Adjunctive therapy with glucocorticoids should also be initiated in immunosuppressive doses to decrease the production of inhibitory anti-ADAMTS13 antibodies.

\section{Case}

A 57-year old male was admitted to our hospital with a several day's history of weakness, prostration and occurrence of dark urine. Laboratory tests from local laboratory revealed anemia and thrombocytopenia. His past medical history and family history were unremarkable.

A physical examination on admission revealed icteric conjunctiva, hematoma on his right lower leg and neurological abnormalities presented as mild headache, disorientation and expressive aphasia. The temperature was $36.7^{\circ} \mathrm{C}$, the blood pressure $100 / 60 \mathrm{~mm} \mathrm{Hg}$, the pulse was 82 beats per minute and the respiratory rate 18 breaths per minute. The lungs were clear and the abdomen was not distended, with any tenderness on palpation or hepatosplenomegaly. There was no swelling in the legs.

Laboratory findings revealed hemolytic anemia with hemoglobin level $67 \mathrm{~g} / \mathrm{L}$; hematocrit $17.3 \%$; reticulocyte count $4.7 \%$; total bilirubin $70.7 \mathrm{umol} / \mathrm{L}$; indirect bilirubin $54.1 \mathrm{umol} / \mathrm{l}$. Aspartate aminotransferase (AST) was $90 \mathrm{U} / \mathrm{L}$, alanine aminotransferase (ALT) $36 \mathrm{U} / \mathrm{L}$, lactate dehydrogenase (LDH) $2170 \mathrm{U} / \mathrm{L}$. There was a significant thrombocytopenia with platelet count $14 \times 10^{9} / \mathrm{L}$. There were also signs of renal damage with blood urea nitrogen 17.0 $\mathrm{mmol} / \mathrm{l}$, serum creatinine $146 \mathrm{umol} / \mathrm{l}$, total serum protein $52 \mathrm{~g} / \mathrm{l}$, albumin $31 \mathrm{~g} / \mathrm{l}$. Urinalysis showed $2+$ blood and 3+ protein Examination of peripheral blood smear revealed 2-3- 


\section{International Journal of Science and Research (IJSR) \\ ISSN (Online): 2319-7064}

Index Copernicus Value (2016): 79.57 | Impact Factor (2015): 6.391

4 schistocytes per high-power field, no erythroblasts were seen. White cells appeared normal and had a normal granulation pattern. Direct and indirect antiglobulin test (Coombs) was negative. Blood coagulation tests (prothrombin time, activated partial thromboplastin time and thrombin time) were all within normal range.

Genetic testing for AHUS genetic panel was negative.

Emergency treatment was started immediately after admission with transfusions of red blood cells, plasmapheresis on daily basis and immunosuppressive treatment with methyl prednisolone $2 \times 250 \mathrm{mg}$ per day. On hospital day 5 the hemoglobin level improved to $105 \mathrm{~g} / \mathrm{L}$ and the platelet count to $68 \times 10^{9} / 1$ and on the hospital day 12 the hemoglobin level was $122 \mathrm{~g} / \mathrm{L}$, the platelet count was $184 \times 10^{9} / \mathrm{L}$ and total bilirubin failed to $15.9 \mathrm{umol} / 1$. As the serum glycaemia grew progressively, Insulin rapid in low doses was started. The clinical condition improved along with the improvement in laboratory results.

\section{Discussion}

Our patient presented with weakness, prostration, occurrence of dark urine and neurological abnormalities, symptoms which were initial presentation of TTP. Laboratory findings revealed hemolytic anemia and thrombocytopenia that could be associated with Evans syndrome. However, the negative antiglobulin test (Coombs) and the lack of spherocytosis in peripheral blood smear that are present in half the cases of the Evans syndrome, excluded this diagnosis. (9).

Microangiopathic hemolytic anemia warrants consideration when schistocytes are seen in a patient with hemolysis (7). Severe hypertension disseminated intravascular coagulation, sepsis, and cancer can cause microangiopathic hemolytic anemia and thrombocytopenia, but there was no evidence of these conditions in our patient. Hemolytic-uremic syndrome (HUS) is a thrombotic microangiopathy that arises when shiga toxin-secreting strains of Escerichia coli, on occasion, Shigelladysenteriae induce endothelial damage that leads to bloody colitis and, subsequently kidney injury; these features were also not present in this case. (8). Atypical hemolytic syndrome (aHUS) is considered a complementmediated form of thrombotic microangiopathy. It is a genetic disease associated with a mutation in CFHR1 (Complement Factor H Related 1) coding gene. AHUS genetic panel was performed in our patient and the result was negative.

TTP can be categorized into 2 major forms, hereditary TTP and acquired TTP (10). The former is caused by mutations of the ADAMTS13 gene. The latter may be idiopathic or nonidiopathic. Idiopathic TTP may result from autoantibodies that inhibit ADAMTS13 function. Nonidiopathic TTP is secondary to other conditions such as hematopoietic stem cell transplantation, certain drugs, infections, other autoimmune diseases, cancers, and so on. An ADAMTS13 activity level that is less than $10 \%$ is highly suggestive of a diagnosis of TTP. Three tests are commonly performed to confirm the diagnosis of TTP: assays for ADAMMTS13 activity, ADAMTS13 inhibition, and antiADAMMTS13 antibodies. The availability of ADAMTS13 testing has helped confirmed the clinical diagnosis of TTP. (11) However, these tests have not been performed in our laboratory.

According to clinical and laboratory findings (four out of the pentad of symptoms for TTP: thrombocytopenia, microangiopathic hemolytic anemia, neurologic abnormalities, and renal disease) a prompt initiation of treatment was started with plasma exchange and high doses of glucocorticoids with clinical and laboratory improvement within two weeks. Prompt initiation of the management of TTP is an important factor in improved patient survival. Prior to plasma exchange, the mortality rate approaches ninety percent whereas with treatment, mortality reaches twenty percent (12).Plasma exchange therapy depletes the circulation level of autoantibody to ADAMTS13 and very high molecular weight von Willebrand factor multimeres along with replacement of the missing protease. TTP is a medical emergency that is almost always fatal if exchange plasmapheresis is not initiated early.

\section{References}

[1] George JN, Nester CM. Syndromes of thrombotic microangiopathy. N Engl J Med 2014; 371:654-666.

[2] Brain MC. An acute pleiochromic anemia with hyaline thrombosis of the terminal arterioles and capillaries (Moschowitz 1925). Thrombosis and Haemostasis 1978, vol.40, no.1, 9-10.

[3] Zheng XL, Salder JE. Pathogenesis of thrombotic microangiopathies. Annu Rev Pathol 2008; 3:247-277.

[4] Lotta LA, Garagiola I, Palla R, Cairo A, Peyvandi F. ADAMTS13 mutations and polymorphisms in congenital thrombotic thrombocytopenic purpura. Hum Mutat.2010 Jan;31(1):11-9.

[5] Esparza-Gordillo J, Jorge EG, Garrido CA, Carreras L, LopezTrascasa M, Sanchez-Corral P, de Cordoba SR. Insights into hemolytic uremic syndrome: segregation of three independent predisposition factors in a large, multiple affected pedigree. Mol Immunol. 2006;43:1769-75.

[6] Booth KK, Terrell DR, Vesely SK, George Jn. Systemic infections mimicking thrombotic thrombocytopenic purpura. Am J Hematol 2011;86:743-751.

[7] Ariceta G, Besbas N, Johnson S, Karpman D, Landau D, Licht C, Loirat C, Pecoraro C, Taylor CM, Van de Kar N, Vandewalle J, Zimmerhackl LB, Zimmerhackl LB. Guideline for the investigation and initial therapy of diarrhea-negative hemolytic uremic syndrome. Pediatr Nephrol. 2009;24:68796.

[8] George JN. How I treat patients with thrombotic thrombocytopenic purpura. Blood 2010; vol.116:4060-4069.

[9] Michael M, Chanet V, Dechartres A. The spectrum of Evans syndrome in adults: new insight into the disease based on the analysis of 68 cases. Blood 2009; 3167-3172.

[10] Zheng XL. ADAMTS13 testing: why bother. Blood 2010; 115:1475-1476.

[11] Peyvandi F, Lavoretano S, Palla R.ADAMTS13 and antiADAMTS13 antibodies as markers for recurrence of acquired thrombotic thrombocytopenic purpura during remission. Haematologica 2008; 93(2):232-239.

[12] Ruggenenti P, Galbusera M, Cornejo RP, Bellavita P, Remuzzi G. Thrombotic thrombocytopenic purpura: evidence that infusion rather than removal of plasma induces remission of the disease. Am J of Kidney Diseases 1993; vol.21 no $3: 314-318$

\section{Volume 6 Issue 12, December 2017}

